\title{
Functional differences in upper limb movement after early and chronic stroke based on kinematic motion indicators
}

\author{
Monika Blaszczyszyna, Agnieszka Szczesna ${ }^{\mathrm{b}}$, Jozef Oparac, Mariusz Konieczny' ${ }^{\mathrm{a}}$, Pawel Pakosz ${ }^{\mathrm{a}}$, Stefan Balko ${ }^{\mathrm{d}}$
}

\begin{abstract}
Aims. The main purpose of this study was to determine the changes in kinematic parameters of ischemic stroke affected upper limbs, during simple functional activity, to determine the most relevant changes.

Methods. The OptiTrack system was used for motion capture. To determine upper extremity function in Activities of Daily Living (ADL) tasks. During particular phases, the following matrices were chosen: mean and peak speed, normalized movement unit, normalized jerk and phase movement time. The chosen matrices represent the speed and smoothness profile of end-point data. The the arm-trunk compensation was also taken into consideration. Twenty stroke patients, in early (G1 from 1 to 3 months after stroke) and chronic stage (G2 from 6 months to 1 year), were studied. The large and small cylinder forward and back transporting phases were evaluated.

Results. The most significant differences between groups G1 and G2 were in mean and peak speed of the forward transport of the large and small cylinders for the paretic limb. Significant differences were also found for the smoothness (measured by movement unit, mean and peak speed and jerk) where the G2 group had a rougher motion. There were also differences in arm-trunk compensation in the frontal plane.
\end{abstract}

Conclusion. The variables used in the study showed applicability in assessing kinematic parameters in both the early and chronic period after stroke.

Key words: stroke, kinematic analysis, upper limb, functional activities

Received: December 28, 2017; Accepted with revision: September 27, 2018; Available online: October 11, 2018 https://doi.org/10.5507/bp.2018.061

${ }^{a}$ Faculty of Physical Education and Physiotherapy, Opole University of Technology, 45-758 Opole, Prószkowska 76, Poland 'Institute of Informatics, Silesian University of Technology, 44-100 Gliwice, Akademicka 16, Poland

'Academy of Physical Education, ul. Mikolowska 72a, 40-065 Katowice, Poland

${ }^{d}$ Department of Physical Education and Sport, Faculty of Education, J.E. Purkyne University, Usti nad Labem, Czech Republic Corresponding author: Monika Blaszczyszyn, e-mail: mblaszczyszyn@wp.pl

\section{INTRODUCTION}

Despite great progress in the treatment of vascular diseases, stroke is still the leading cause of long-term disability in the world ${ }^{1-3}$. Many stroke survivors experience complex neurological deficits that impair movement quality, resulting not only from motor problems, but also from cognitive and behavioral problems ${ }^{3}$.

The National Stroke Association Survey found that $87 \%$ of persons after stroke had ongoing motor problems, $54 \%$ had trouble walking, $52 \%$ had trouble with hand movements, and $58 \%$ experienced spasticity ${ }^{46}$.

One of the most important problems after stroke is functional limitation of the upper limb $b^{6-8}$. Careful investigation $\mathrm{s}$ of these motor problems and their causes may contribute to the implementation of appropriate rehabilitation processes with positive impact on functional improvement in the acute and chronic period ${ }^{9-11}$.

Clinical scales most commonly used to assess motor function of the paretic upper limb are largely subjective and in many cases not sensitive, especially in patients with good clinical status or with only slight motor improvement ${ }^{12}$.
Kinematic indices are the starting point for proposed objective indicators for functional assessment of the upper limb in people with motor disorders as a result of neurological diseases ${ }^{13}$. Optical motion capture systems with passive markers are the most widely used technologies as a gold standard in kinematic analysis because of their high accuracy and reliability ${ }^{14}$. They can provide kinematic measures of movement duration, accuracy and smoothness, or dynamic measures of forces and work expended during therapy ${ }^{15-18}$.

Upper-extremity point-to-point reaching movement related kinematic metrics can be classified into two categories: end-point (hand) kinematic metrics and joint kinematic metrics ${ }^{13,19}$.

Motion data for upper limbs may be collected by examining the various representative Activities of Daily Living (ADL) tasks. One such activity is the movement of transporting and lowering of objects at different heights, which is necessary in many ADL.

The main purpose of the study was to determine the changes in kinematic parameters of ischemic stroke affected upper limb during simple functional activities to determine the most relevant changes to the movement. 


\section{METHODS}

\section{Participants}

A total of 34 stroke patients residing in the neurological rehabilitation ward were qualified for the study. Assessment of clinical status was made on the day of admission to the neurological rehabilitation department. All patients after ischemic stroke (confirmed by CT) who had been identified for the study were divided into two groups according to the time since the stroke: G1 persons in the early period, from 1 to 3 months after stroke, and G2 persons in the chronic period, from 6 months to 1 year. The following inclusion criteria were adopted: first-ever ischemic stroke, retention of the hand to a degree to hold the objects used in the study, limited quality or speed of performance, spasticity less than or equal to 2 according to the modified Ashworth scale, ability to understand instructions and active participation in tasks. Patients with cognitive impairment, severe aphasia, visual impairment, behavioral disorders, disturbances of cognitive functions (MMSE<24) or joint stiffness were excluded. Participants were assessed using functional scales National Institute of Health Stroke Scale (NIHSS), Functional Indicator "Repty" (WFR) is a Polish FIM modification, and FuglMeyer Motor Assessment (FMA - upper limb). 14 patients, out of 34 , were not analyzed, including 4 patients due to the lack of accuracy in registration of the study, 2 patients with outlier results were rejected, 1 person after amputation of the lower limb, 4 people due to accompanying diseases that prevented participation in the study, 3 people due to lack of muscular strength. The remaining 20 patients - 12 in the early period and 8 in the chronic period were analyzed according to the inclusion criteria for the study. Detailed kinematic analysis revealed significant differences between patient groups.

All participants received information about the experimental procedures to be carried out and they gave their

Table 1. Clinical characteristics of participants.

\begin{tabular}{lcc}
\hline Variables & $\mathrm{G} 1(\mathrm{n}=12)$ & $\mathrm{G} 2(\mathrm{n}=8)$ \\
\hline Gender male/female & $7 / 5$ & $6 / 2$ \\
Mean age $\pm \mathrm{SD}$ (years) & $62 \pm 11.6$ & $64 \pm 12.7$ \\
Paresis R/L & $8 / 4$ & $6 / 2$ \\
Time after stroke $\pm \mathrm{SD}$ (days) & $63.2 \pm 19.7$ & $285.5 \pm 39.6$ \\
Stroke type & $\mathrm{n}$ & $\mathrm{n}$ \\
PACI & 7 & 5 \\
TACI & 4 & 2 \\
LACI & 1 & 1 \\
Arterial hypertension & 9 & 6 \\
Diabetes & 1 & 3 \\
Atrial fibrillation & 2 & 1 \\
Lipid disorders & 6 & 4 \\
NIHSS total score & $4(2-5)$ & $4(2-5)$ \\
WFR \pm SD & $89.62 \pm 9.08$ & $90.37 \pm 10.06$ \\
FMA(upper limb) $\pm S D$ & $48.3 \pm 11.0$ & $49.4 \pm 10.5$ \\
\hline
\end{tabular}

PACI - parietal anterior cerebral ischemia, TACI - total anterior cerebral ischemia, LACI - lacunar cerebral ischemia, rt-PA - recombinant tissue plasminogen activator, SD - standard deviation written consent to participate in the study. The research project was approved by the Bioethics Committee at the Opole Medical Chamber No. 215 of March 25, 2015. Table 1 describes the characteristics of the participants.

\section{Instrument}

The OptiTrack optoelectronic motion analysis system based on passive, reflective markers allows for a comprehensive assessment of the kinetic and kinematic parameters of any motion. Eight infrared cameras capture markers movement at a maximum resolution of $832 \times 832$ pixels and up to 250 frames per second.

\section{Experimental procedure}

The participants carried out the task of successively raising large and small cylinders. After taking a seat with lower limbs flexing to 90 degrees at the knee and hip joint, feet placed on the ground, hands resting on the edge of the table, fingers 2-5 were on the table, thumbs were under the table. Objects were placed on the table with additional markers. The patients performed two tasks of lifting a small (12 mm diameter cylinder, $5 \mathrm{~cm}$ long, weighing $190 \mathrm{~g}$ ) and big (34 $\mathrm{mm}$ diameter, $7 \mathrm{~cm}$ long, weighing $450 \mathrm{~g}$ ) cylinder.

The analysis specifies two phases of motion: lifting and lowering. The lift phase (called forward transporting, FT) analysis started from the moment the cylinder was held firmly and the uptake was up and ended when the highest point was reached. The lowering phase (called back transporting, BT) started at the moment the initiation of the lowering motion of the cylinder ended when the cylinder contacted the table top.

Fig. 1. presents the complete set of markers included in the protocol. The protocol describes several functional activities, namely: drinking from a glass, lifting a small and a large cylinder, closing and unscrewing a jar, removing a clip, combing hair, drawing lines, similar to the Frenchay Arm Test (FAT) reference, and the protocol provided for a broader analysis comparative kinematic parameters, including joint angles. Included here are only lifting and lowering a small and a large cylinder.

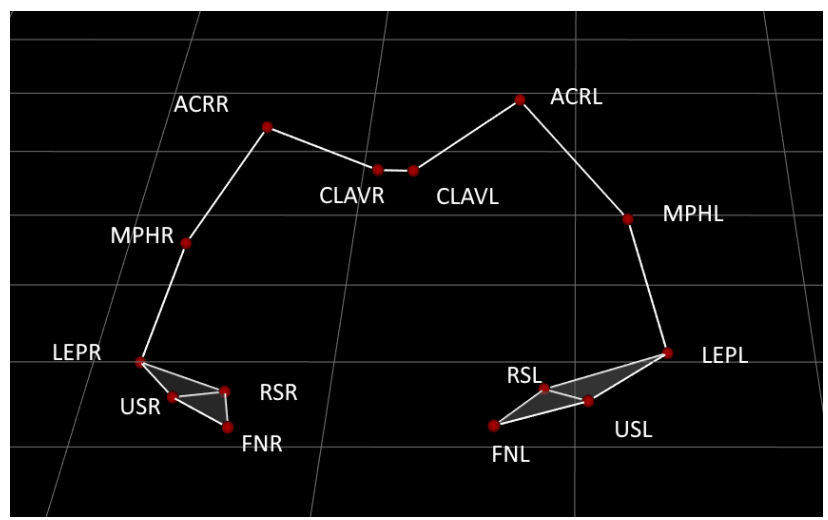

Fig. 1. Upper body marker placement schema. Markers were located on both upper limbs (right R/L left), CLAVR/L - clavicular heads, ACRR/L - acromion process, MPHR/L - middle part of the humeri, LEPR/L - lateral epicondyle, RSR/L - radial styloid, USR/L - ulnar styloid, FNR/L - index finger nails. 
a. Data Preparation and Analysis

Motion was measured by the OptiTrack system during forward transporting of the small and big cylinder. The presented end-point kinematic analysis is based on the $3 \mathrm{D}$ trajectory of the marker placed on the index finger of the left (FNL) and right hand (FNR). The analyzed task sequence starts with preparing and grasping the cylinder, lifting the cylinder FT over the patient's head, BT and putting down the cylinder in a designated place on the table. In the analysis only FT and BT phases were considered. Data was captured with the frequency $100 \mathrm{~Hz}$.

\section{b. Quantitative Kinematic Indices}

To analyze upper extremity ADL movement during particular phases, the following matrices were chosen: mean and peak speed, normalized movement unit, normalized jerk and phase movement time. The chosen matrices represent the speed and smoothness profile of end-point data (see classification in ref. 13). Additionally the arm-trunk compensation index was analyzed in each plane (traverse, frontal and sagittal).

Movement time is a time in seconds required to perform a task successfully. Movement mean speed is computed based on the mean value of temporary marker speed and is based on marker 3D displacement and time during the phase. The movement unit is the number of acceleration peaks in kinematic data and quantitative measure movement smoothness. Fewer peaks represent fewer periods of acceleration and deceleration, making a smoother movement. We used a normalized movement unit by total displacement of the marker. A normal reaching movement has only one peak in the velocity profile of the hand movement ${ }^{20}$. In movement disorders, the velocity peak number increases, resulting in a jerkier movement.
The next index to measure movement smoothness is jerk. The jerk is the third time derivative of $3 \mathrm{D}$ point (marker) displacement: $J(t)=\frac{d^{3} x}{d t^{3}}$, where $x$ is a marker position vector and $t$ is the time variable. The jerk is the rate of the acceleration change in time, and some studies indicate that humans by nature tend to minimize the jerk parameter over the duration of the reaching movement ${ }^{21}$. The smoothest motion has the lowest jerk values. The normalized jerk was introduced in a way where the influence of movement $\frac{T^{3}}{L}$ length and duration is removed by multiplying by the factor where $T$ is movement duration and $L$ total marker displacement ${ }^{22}$. Therefore, normalized jerk is a unit-free variable and can be compared across different persons in a group. We used the mean value of normalized jerk value:

$N J=\operatorname{mean}\left(J_{i}\right)$, where $\mathrm{Ji}$ are normalized discrete jerk values for samples: $J_{i}=\sqrt{J_{x, i}^{2}+J_{y, i}^{2}+J_{z, i}^{2}}$.

Trunk compensation was denoted by the arm-trunk compensation index in each plane (frontal, transverse and sagittal). The index is computed based on the distance (1) between the first and last position in a phase (FT and BT) and is expressed as the ratio between the difference of the distance of the index finger marker (FNL or FNR) and a virtual marker which represented the sternum marker, to the distance of the index finger marker $A R C=1-\frac{l_{F N L / F N R}-l_{\text {mean }(C L A V R, C L A V L)}}{l_{F N L / F N R}}$. A lower ARC value indicates lower trunk displacement and lower trunk compensation. The virtual sternum marker is computed as the average marker of CLAVR and CLAVL. The ARC index can take values between $\langle 0,1\rangle$ so can be easily used to compare between subjects.

Fig. 2. illustrates an example of calculating the ARC index in the frontal plane during the lifting phase of a large cylinder for a G1 patient.

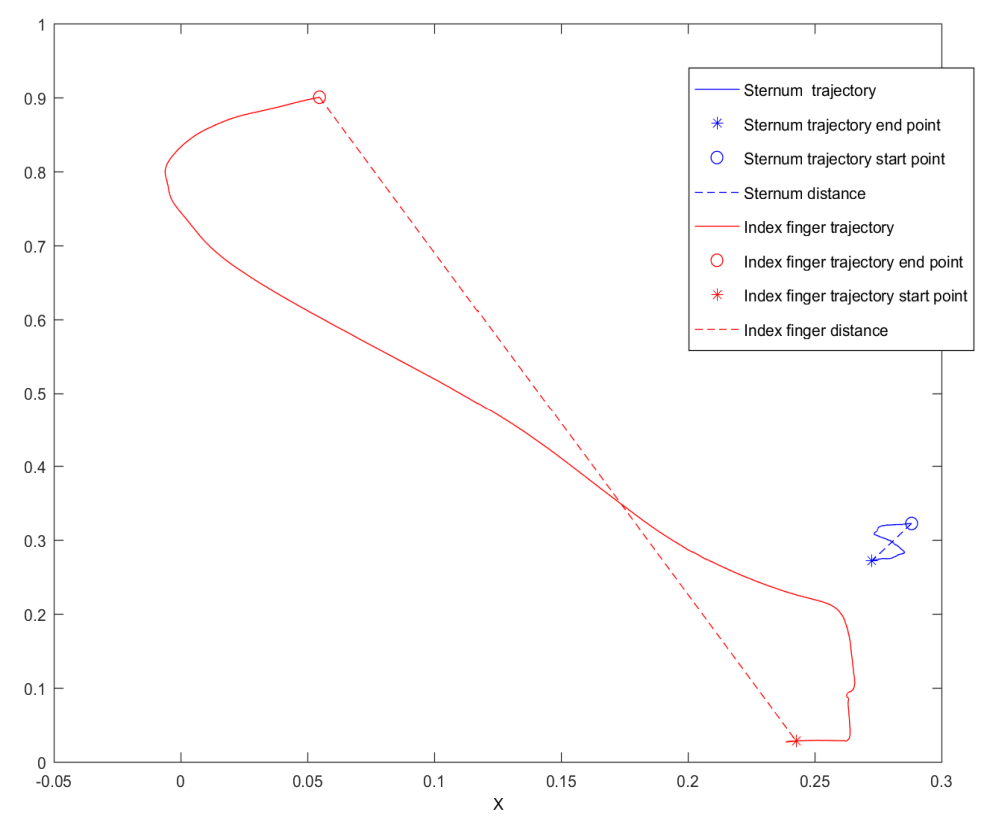

Fig. 2. The ARC index in the frontal plane during the lifting phase of a large cylinder for a G1 patient. 


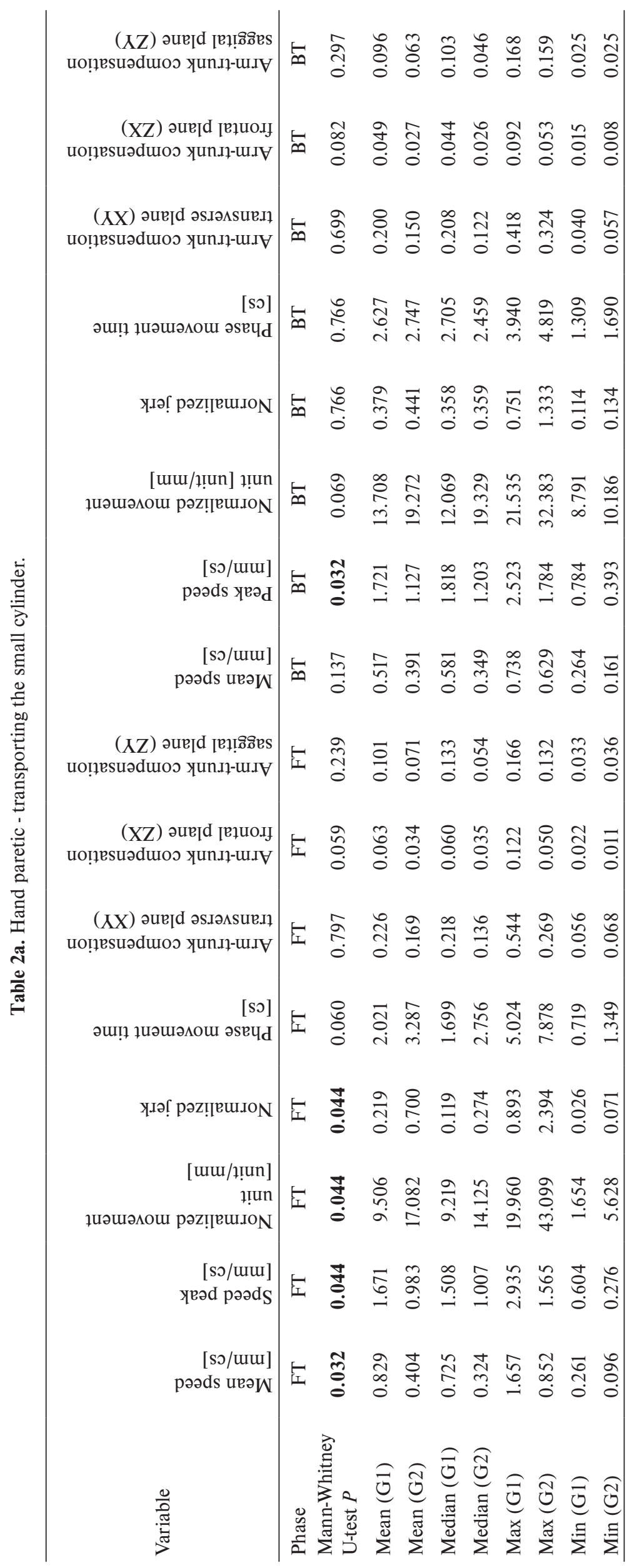




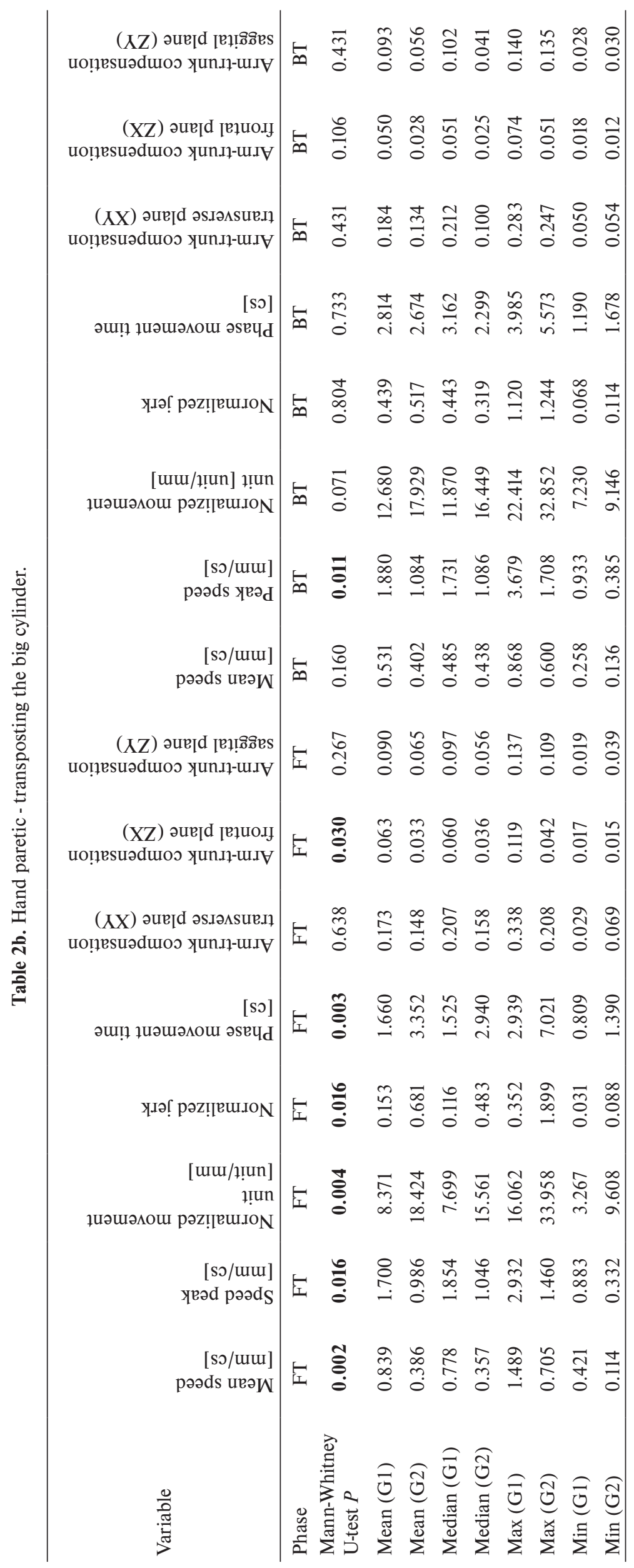









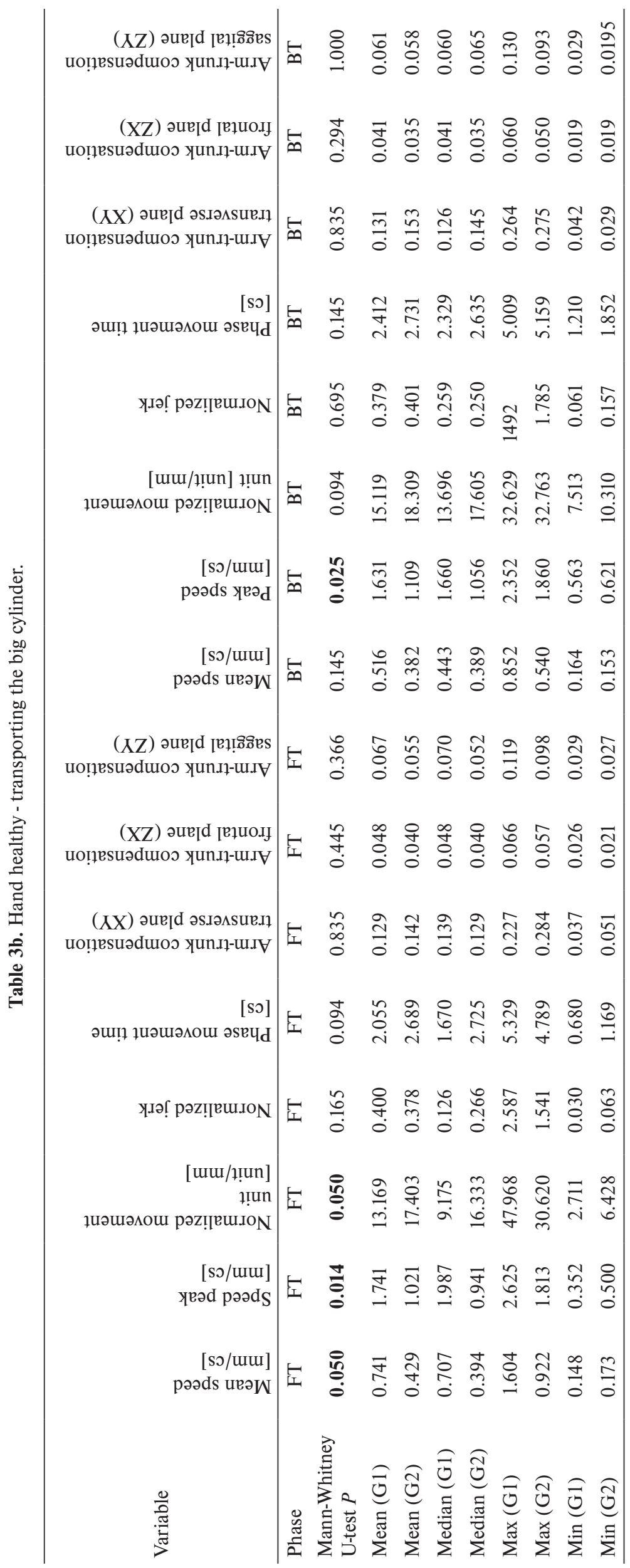




\section{Statistical Analysis}

Nonparametric Mann-Whitney U-test was used for comparisons of kinematic indexes between groups p-values equal to or less than 0.05 were statistically significant.

\section{RESULTS}

The subjects had mild to moderate disability assessed by the WFR and FMA-UE. All respondents were right-handed. There was no statistical difference between paretic side for G1 and G2 groups (Mann-Whitney U-test, $P=0.26$ ) or gender (MannWhitney U-test, $P=0.13$ ).

The all results are summarized in Tables 2 a,b and 3 a,b.

The main comparison was based on the G1 and G2 difference in both healthy and paretic limbs.

The most significant differences between groups G1 and $\mathrm{G} 2$ were for the results of the forward transporting of the small and big cylinders for the paretic limb. Significant differences were shown for the same variables for the small and big cylinder. For the FT, they are mean speed and peak speed (where during the movement group G1 reached higher speeds). Significant differences were also found for the smoothness (jerk and movement unit) where the $\mathrm{G} 2$ group had a jerkier motion. There were differences in arm-trunk compensation in the frontal plane.

The G2 group had a longer cylinder lifting time and statistically significantly longer execution time for this unit phase, which could have been affected by the larger size of the object. Such differences are not found in the phase of BT, where times are very similar. The same relationship can be observed for healthy limb movement. The observations concerning the performance of the same activities with the healthy limb are very interesting. There were also statistically significant differences in the phase FT for speed estimators: mean speed and peak speed. The differences are also present for smoothness measurements for the FT: normalized movement unit and jerk with the same tendency. That is, a higher speed value was achieved in the G1 group and less smoothness in the G2 group. However, there was no significant difference in the arm-trunk compensation variable. It should also be noted that with the paretic limb G2 patients had less arm-trunk compensation in each plane (lower compensation factor). This applied both to the forward transporting and back transporting phases. Such a relationship was not observed for a healthy limb movement, where the values of the compensation factors are very close.

\section{DISCUSSION}

The authors performed a comparative analysis of patients in early and chronic stroke. The kinematic evaluation of motion was performed on the basis of the following variables: mean speed, normalized movement unit, normalized jerk, phase movement time and armtrunk compensation index. Quantitative measures of human motion quality are important in the rehabilitation field for expressing the outcomes during rehabilitation treatments and assessing their efficacy, discriminating between healthy and pathological conditions, and for helping in the treatment decision making ${ }^{23}$. Kinematic indexes are quantitative measures based on the 3D trajectory of movement and provide information about the movement's quality with respect to coordination, smoothness and other functional characteristics ${ }^{13,22}$, related to movement efficiency, speed, and accuracy, possibly revealing different working mechanisms of recovery after stroke ${ }^{24}$.

The positions of markers are captured by optical motion capture system OptiTrack. All presented indices are based on 3D trajectory. Based on the documentation of the OptiTrack system, the position error is negligible, because the $3 \mathrm{D}$ location of markers can be resolved with sub-millimeter accuracy for the used configuration.

Evaluated variables showed differences in the evaluated activities, and statistical significance was observed mainly in the transporting phase. Participants in the chronic period had significantly worse results for the variables tested in the assessment of both the paretic and the healthy limb. The results obtained may suggest that, despite access to rehabilitation, post-stroke patients exhibit significant impairment in motor activity. This may be due to the fact that there are many cognitive, behavioral or specific compensatory disorders associated with the development of motor-specific synergies characteristic for this group of patients, because one still cannot clearly determine how the recovery process occurs in patients. Most hypotheses are based on the theory of reorganization of brain cortical structures, the analysis of the cerebral cortical structure of cerebral hemispheres that can be used to understand the post-stroke recovery phenomena, both in patients with good recovery (positive reorganization) and those whose recovery process is slow or limited (negative reorganization) $\left(\right.$ ref $\left.^{25}\right)$. This theory points to the need for improvement in the form of bilateral exercises involving both the healthy and the impaired cerebral hemispheres ${ }^{26,27}$.

Performance after stroke of reaching movements in stroke patients is characterized by a pathological synergy that manifests as disruption in gross extensor movement called extensor synergy (shoulder extension and adduction combined with elbow extension) or a gross flexor movement called flexor synergy (shoulder flexion and abduction combined with elbow flexion) (ref. ${ }^{28}$ ).

Moreover, the neuromuscular coordination of the body segments involved in the transporting movement is disturbed, which contributes to individual compensation based on the most efficient cells and the reduction of the most impaired cells, which over time contributes to the development of abnormal motor patterns. Patients in the chronic stroke period exhibited much worse results in the evaluated activities than patients in the early stages, both in healthy and paretic limbs.

Our results show that kinematic parameters such as mean speed, normalized movement unit, normalized jerk, and phase movement time are sensitive to qualitative and quantitative changes in the affected limb, in both early and chronic periods. Thus, they can be, an appropriate 
tool for assessing the progress of rehabilitation and its planning process. They revealed significant differences in the assessed phases of movement and in the study groups. This study can contribute to a better understanding of the changes occurring in upper limb motility as a result of stroke in relation to simple functional activities. Applied motion kinematic parameters facilitate clinical examination of patients in terms of motion analysis and can be valuable not only to better plan the rehabilitation process but also in bioengineering ${ }^{29}$.

There are a large number of studies showing the differences in ADL activity in stroke survivors compared to healthy individuals of similar age. They clearly indicate that post-stroke patients use a different type of motor strategy in performing ADL, as a result of damage to the brain structures and consequently observed movement limitations. It is interesting that these changes affect both the healthy and paretic limb $b^{30}$.

Tran et al. ${ }^{24}$ pointed out, there is no consensus on the most appropriate outcome measures nor on the kinematic parameters that should be used. Kinematic measures have become more important in the evaluation process, because motor performance can be highlighted through the analysis of the kinematic parameters recorded ${ }^{23,24}$. It should be also noted that such procedures are expensive with unfortunately limited access.

\section{CONCLUSION}

The variables used in the study showed sensitivity in assessing kinematic parameters both in early and chronic stroke. They can be used as a tool to evaluate the results of rehabilitation and planning the therapeutic process. They indicate which kinematic parameters during a specific functional activity are impaired and therefore what are the needs for an effective process of improving the upper limb function of a particular person, both in the early and chronic period. In addition, the subjects achieved more speed and smoothness during the purposeful motion. Disorders in both limbs may indicate the need for bilateral exercises involving the healthy and impaired cerebral hemisphere.

\section{Limitations}

The pilot study revealed the imperfections associated with the patient implantation procedure. The original protocol included too few markers and consequently failed to achieve several significant goals, for example lack of ability to analyze rotational movements. Certainly the test should be repeated with a larger sample size and more detailed marking.

Acknowledgments: This work was partial supported by a statute project of the Silesian University of Technology, Institute of Informatics (BK/RAU-2/2018) (A.Szczęsna). Author contributions: MB, ASZ, literature search; MB, ASZ, JO, SB: manuscript writing; MB, PP, MK: data collection; ASZ: methods implementation, data analysis.
Conflic of interest statement: The authors state that there are no conflicts of interest regarding the publication of this article.

\section{REFERENCES}

1. Feigin VL, Forouzanfar MH, Krishnamurthi R, Mensah GA, Connor M, Bennett DA, Moran AE, Sacco RL, Anderson L, Truelsen T, O'Donnell M, Venketasubramanian N, Barker-Collo S, Lawes CM, Wang W, Shinohara Y, Witt E, Ezzati M, Naghavi M, Murray C. Global and regional burden of stroke during 1990-2010: findings from the Global Burden of Disease Study 2010, The Lancet 2014;383,9913:245-55.

2. Roger VL, Go AS, Lloyd-Jones DM, Adams RJ, Berry JD, Brown TM, Carnethon MR, Dai S, de Simone G, Ford ES, Fox CS, Fullerton HJ, Gillespie C, Greenlund KJ, Hailpern SM, Heit JA, Ho PM, Howard VJ, Kissela BM, Kittner SJ, Lackland DT, Lichtman JH, Lisabeth LD, Makuc DM, Marcus GM, Marelli A, Matchar DB, McDermott MM, Meigs JB, Moy CS, Mozaffarian D, Mussolino ME, Nichol G, Paynter NP, Rosamond WD, Sorlie PD, Stafford RS, Turan TN, Turner MB, Wong ND, Wylie-Rosett J. Heart disease and stroke statistics - 2011 update: a report from the American Heart Association, Circulation 2011;123:18-209.

3. Cardol M, De Jong BA, Van den Bos GA, Beelem A, de Groot IJ, de Haan RJ. Beyond disability: perceived participation in people with a chronic disabling condition. Clin Rehabil 2002;16:27-35.

4. Jones VN. The forgotten survivor. Stroke Smart. Available from: http://www.strong.org/site/PageServer?pagename 5 SS_MAG_ so2006_feature_forgot; 2006.

5. Murphy MA, Willen C, Sunnerhagen KS., Kinematic variables quantifying upper-extremity performance after stroke during reaching and drinking from a glass. Neurorehabil Neural Repair 2011;25:71-80.

6. Lai SM, Studenski S, Duncan PW, Perera S. Persisting consequences of stroke measured by the Stroke Impact Scale. Stroke 2002;33:18404.

7. Broeks JG, Lankhorst GJ, Rumping K, Prevo AJ. The long-term outcome of arm function after stroke: results of a follow-up study. Disabil Rehabil 1999;21(8):357-64.

8. Johansson A, Mishina E, Ivanov A, Bjorklund A. Activities of daily living among St Petersburg women after mild stroke. Occup Ther Int 2007;14(3):170-82.

9. Nichols-Larsen DS, Clark PC, Zeringue A, Greenspan A, Blanton S. Factors influencing stroke survivors' quality of life during subacute recovery, Stroke 2005;36(7):1480-4.

10. Langhorne $P$, Coupar F, Pollock A. Motor recovery after stroke: a systematic review. Lancet Neurol 2009;8(8):741-54.

11. Page SJ, Gater DR, Bach YRP. Reconsidering the motor recovery plateau in stroke rehabilitation. Arch Phys Med Rehabil 2004;85(8):137781.

12. Ozturk A, Tartar A, Ersoz Huseyinsinoglu B. Kinematic analysis of upper extremity motor function after stroke, in: TIPTEKNO (TıpTeknolojileri Ulusal Kongresi) 25-27 September, 2014, Kapadokya.

13. de los Reyes-Guzmán A, Dimbwadyo-Terrer I, Trincado-Alonso F, Monasterio-Huelin F, Torricelli D, Gil-Agudo A., Quantitative assessment based on kinematic measures of functional impairments during upper extremity movements: A review. Clin Biomech (Bristol, Avon);29(7):719-27.

14. Ozturk A, Tartar A, Huseyinsinoglu BE, Ertas AH. A clinically feasible kinematic assessment method of upper extremity motor function impairment after stroke. Measurement 2016;80:207-16.

15. Zollo L, Rossini L, Bravi MG, Magrone G, Sterzi S, Guglielmelli E., Quantitative evaluation of upper-limb motor control in robot-aided rehabilitation. Med Biol Eng Comput 2011;(10):1131-44.

16. Papaleo E, Zollo L, Spedaliere L, Guglielmelli E. Patient-tailored adaptive robotic system for upper-limb rehabilitation, in: 2013 IEEE International Conference on Robotics and Automation, (ICRA), IEEE,2013:3860-5.

17. Murphy MA, WilLen KSC, Sunnerhagen KS. Kinematic variables quantifying upper extremity performance after stroke during reaching and drinking from a glass. Neurorehabil Neural Repair 2011;25(1):7180 . 
18. Patterson TS, Bishop MD, McGuirkTE, Sethi A, Richards LG. Reliability of upper extremity kinematics while performing different tasks in individuals with stroke. J Mot Behav 2011;43:121-30.

19. Jaspers E, Feys H, Bruyninckx H, Molenaers G, Desloovere K. The Arm Profile score: a new summary index to assess upper limb movement pathology. Gait Posture 2011;34(2):227-33.

20. Chang JJ, Wu TI, Wu WL, Su FC. Kinematical measure for spastic reaching in children with cerebral palsy. Clinical Biomech 2005;20:381-8.

21. Hogan N. An organizing principle for a class of voluntary movements. Soc Neuroscience 1984;11:2745-54

22. Teulings HL, Contreras-Vidal JL, Stelmach GE, Adler CH. Parkinsonism reduces coordination of fingers, wrist, and arm in fine motor control. Exp Neurol 1997;146:159-70.

23. Krebs HI, Aisen ML,Volpe BT, Hogan N. Quantization of continuous arm movements in humans with brain injury. National Acad Sciences 1999;96:4645-9.

24. Tran VD, Paolo Dario P, Mazzoleni S. Kinematic measures for upper limb robot-assisted therapy following stroke and correlations with clinical outcome measures: A review. Medical Engineering and Physics 2018;53:13-31.
25. Panarese A, Pirondini E, Tropea P, Cesqui B, Federico Posteraro F, Micera S. Model-based variables for the kinematic assessment of upper-extremity impairments in post-stroke patients. Journal of NeuroEngineering and Rehabilitation 2016;13:81.

26. Grefkes C, Fink GR. Connectivity-based approaches in stroke and recovery of Function. Lancet Neurol 2014;13:206-16.

27. DeJong SL, Lang CE. Comparison of unilateral versus bilateral upper extremity task. Top Stroke Rehabil 2012;19(4):294-305.

28. Kantak S, McGrath R, Zahedi N. Goal Conceptualization and symmetry of arm movements affect bimanual coordination in individuals after stroke. Neuroscience Letters 2016;626:86-93.

29. Murphy MA, Willén C, Sunnerhagen KS. Responsiveness of Upper Extremity Kinematic Measures and Clinical Improvement During the First Three Months After Stroke. Neurorehabilitation and Neural Repair 2013;27(9):844-53.

30. Reisman DS, Scholz JP. Aspects of joint coordination are preserved during pointing in persons with post-stroke hemiparesis. Brain 2003; 126(Pt 11):2510-27. 